\title{
Status of the Fast Interaction Trigger for ALICE Upgrade
}

\author{
Maciej Slupecki on behalf of the ALICE Collaboration ${ }^{a, *}$ \\ ${ }^{a}$ Helsinki Institute of Physics, \\ P.O. Box 64, 00014 University of Helsinki, Finland
}

E-mail: maciej.slupecki@cern.ch

The Fast Interaction Trigger (FIT) denotes a set of three new detectors being part of the upgrade of ALICE in preparation for Run 3 of the LHC at CERN. To fulfill its physics objectives, ALICE aims to collect in Run 3 all minimum-bias data at the target interaction rate of $50 \mathrm{kHz}$ for $\mathrm{Pb}-\mathrm{Pb}$ and up to $1 \mathrm{MHz}$ for pp collisions. This challenging increase in data rates of almost two orders of magnitude compared with Run 1 and Run 2 requires major improvements in all ALICE subdetectors, especially in their readout. While some of the upgraded ALICE detectors can operate in a continuous readout mode others need the fast trigger, specifically: Transition Radiation Detector (TRD), Charged-Particle Veto (CPV), High Momentum Particle Identification (HMPID), calorimeters and Photon Spectrometer (PHOS). Besides generating triggers for them, the FIT detectors will monitor luminosity, will provide the collision start time for particle identification via the time of flight, will measure the particle multiplicity in the forward direction, the centrality and the event plane, and will participate in diffractive physics measurements.

The FIT consists of the following three detectors: FT0 - a set of two fast Cherenkov arrays specializing in minimum-bias trigger generation and luminosity measurement, located on both sides of the interaction point; FV0 - a large segmented scintillator disk focusing on multiplicity triggers and event plane determination, attached to the FT0 array located opposite to the Muon Spectrometer; and FDD (Forward Diffractive Detector) - also a scintillator located at very high pseudorapidities, concentrating on background monitoring and diffractive physics.

The assembly of FIT detectors is close to completion. All components are on time for installation at ALICE between December 2020 and May 2021 according to the present schedule.

40th International Conference on High Energy physics - ICHEP2020

July 28 - August 6, 2020

Prague, Czech Republic (virtual meeting)

\footnotetext{
*Speaker
} 


\section{Fast Interaction Trigger (FIT) at ALICE}

ALICE physics objectives for LHC Run 3 and 4 [1] require recording all minimum-bias events in $\mathrm{Pb}-\mathrm{Pb}$ collisions. Compared to Run 2, this constitutes an increase of almost two orders of magnitude in terms of the recorded data. The target interaction rate and bunch-crossing time are $50 \mathrm{kHz}$ and $50 \mathrm{~ns}$ for $\mathrm{Pb}-\mathrm{Pb}$, and $1 \mathrm{MHz}$ and $25 \mathrm{~ns}$ for pp collisions. Such extreme running conditions impose stringent requirements on the ALICE subsystems, including the Fast Interaction Trigger (FIT). The functionalities of FIT are the following:

- Instantaneous luminosity measurement with direct feedback to the LHC;

- Trigger generation, especially important for the ALICE subdetectors that cannot acquire data continuously, i.e., Transition Radiation Detector (TRD), Charged-Particle Veto (CPV), High Momentum Particle Identification (HMPID), calorimeters and Photon Spectrometer (PHOS); the following types of triggers will be generated: a minimum-bias trigger with online vertex determination that rejects beam-gas and beam-halo background and provides veto for ultraperipheral $\mathrm{Pb}-\mathrm{Pb}$ collisions, and high-multiplicity or centrality trigger;

- Collision-time determination for the Time-Of-Flight (TOF) detector, especially in events with low charged-particle multiplicities;

- Event-by-event measurement of particle multiplicity, centrality and event plane;

- Participation in diffractive physics measurements.

\subsection{Design and subdetectors}

Figure 1 shows a CAD drawing of the key elements of FIT. They are grouped into three distinct detectors:

\section{- FT0}

The FT0 comprises two modular Cherenkov detector arrays located on both sides of the interaction point (IP): FT0-A and FT0-C. The FT0-A is composed of 24 modules and the FT0-C of 28. Each module has a Microchannel Plate Photomultiplier Tube (MCP-PMT) photosensor (PLANACON ${ }^{\circledR}$ XP85002/FIT-Q), optically coupled with the Dow Corning ${ }^{\circledR} 200$ optical grease to 4 Spectrosil $^{\circledR} 2000$ quartz radiators. The MCP-PMT anode is also divided into 4 matching sectors so that each module delivers 4 independent readout channels/pixels. The sides of the radiators are mirror-covered to reduce light loss and optical cross-talk. The entrance side has black paper glued to it to absorb light generated by particles moving in the opposite direction. To monitor time and amplitude resolution, FT0 is equipped with a laser calibration system, feeding laser pulses to each quartz radiator. The single-pixel time resolution for one minimum ionizing particle (MIP) is 13 ps. FT0 generates a lowlatency minimum-bias trigger, measures the collision time, provides background rejection, and monitors luminosity.

\section{- FV0}

The FV0 is a segmented scintillation detector. FV0 is divided into five rings with progressively 


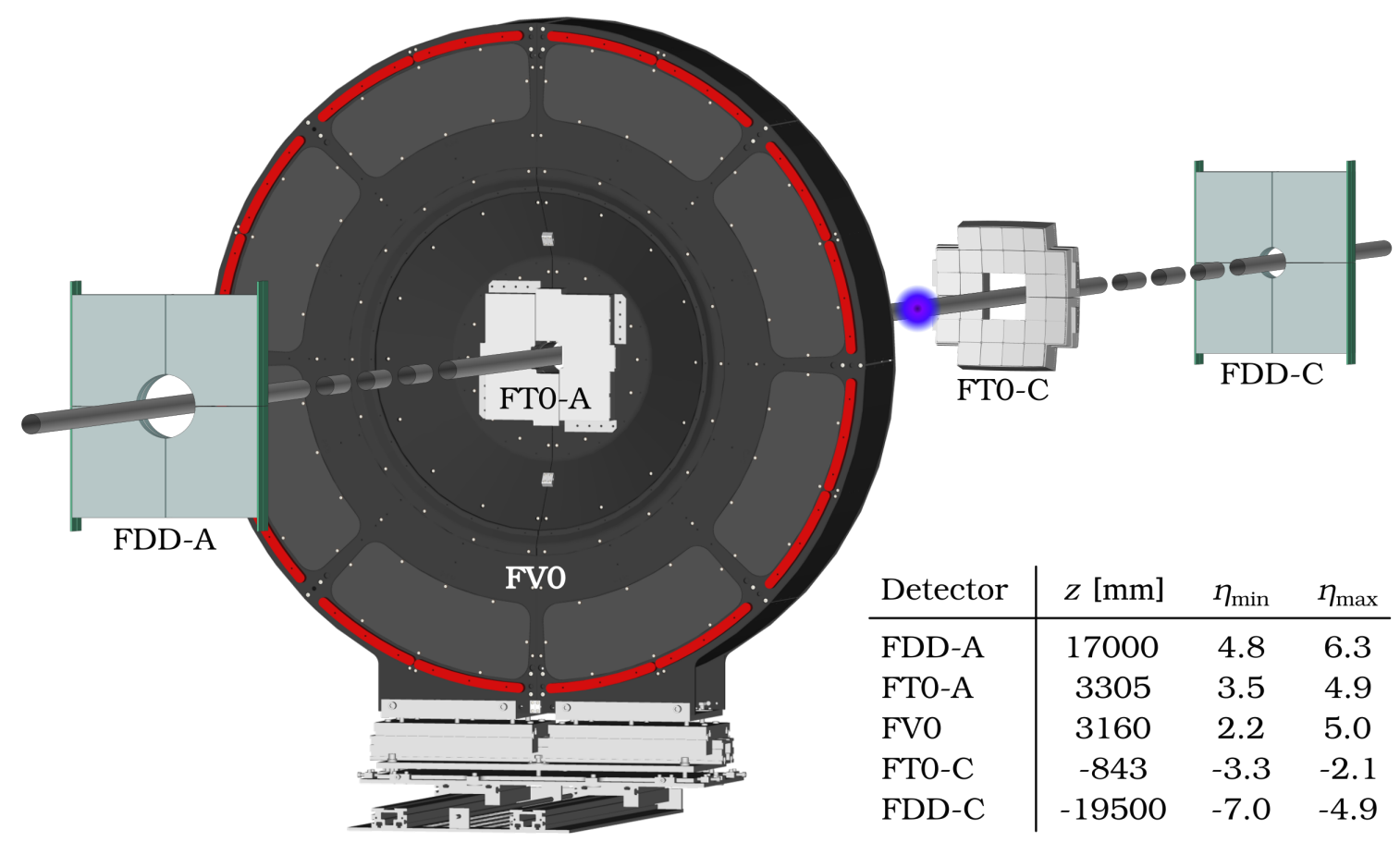

Figure 1: CAD drawing of the key elements of FIT. From left: FDD-A, FV0 (the large circular structure), FT0-A (at the centre of FV0), FT0-C, and FDD-C. The violet sphere marks the interaction point (IP). All detectors are drawn to scale. The actual distances from the IP, measured along the beam direction $(z)$, are listed in the inset together with the pseudorapidity coverage.

increasing radii to ensure similar pseudorapidity coverage of each ring. Azimuthally, the rings are divided into eight sectors forming a total of 40 optically insulated cells made of a 4-cm thick EJ-204 plastic scintillator. Bundles of clear optical fibers, attached to the rear side of each cell, collect and transport light to fine-mesh PMTs (Hamamatsu R5924-70) located beyond the outer radius of the largest ring. By eliminating wavelength shifters, this light collection system reduces the overall pulse length and yields a single-MIP time resolution of 200-300 ps. One cell is read out by a single PMT. Because of its size, each cell of the outermost ring has two photosensors attached. In total, FV0 has 48 readout channels. Thanks to the large active area and good timing, FV0 will contribute to the low-latency multiplicity trigger and will be used to monitor the luminosity and background.

\section{- Forward Diffractive Detector (FDD)}

The FDD consists of two scintillator arrays located on both sides of the IP at a distance of $17 \mathrm{~m}$ and $19.5 \mathrm{~m}$. Each array has a double layer of four azimuthally-divided $2.5 \mathrm{~cm}$ thick BC-420 scintillator cells. Two plastic bars doped with a fast wavelength shifter (NOL-38) collect the light from each cell. The light is then transported by clear-fiber bundles to the remote PMT assembly (Hamamatsu H8409-70). Thanks to its very forward location the FDD will be capable of tagging photon-induced or diffractive processes by recognizing the absence of activity in the forward direction. It will also be an important background-monitoring tool. 


\subsection{Readout electronics}

FIT detectors use the same custom-designed Front-End Electronics (FEE) capable of continuous readout at the bunch-crossing interval of $25 \mathrm{~ns}$. The only detector-specific modifications are minor and reflect differences in the pulse shape of the input signals between the three subdetectors. The dynamic range of FEE charge readout is 0.4:250 MIPs per channel. Reaching the 0.4 MIP threshold at the input was essential because there are no active elements between the detectors and the FEE rack, about 30-50 m away. FIT uses double-shielded, low-attenuation coaxial cables to transmit analogue pulses. Besides the charge integration, FIT FEE derives and digitizes the signal arrival time with a resolution (bin width) of 13.02 ps.

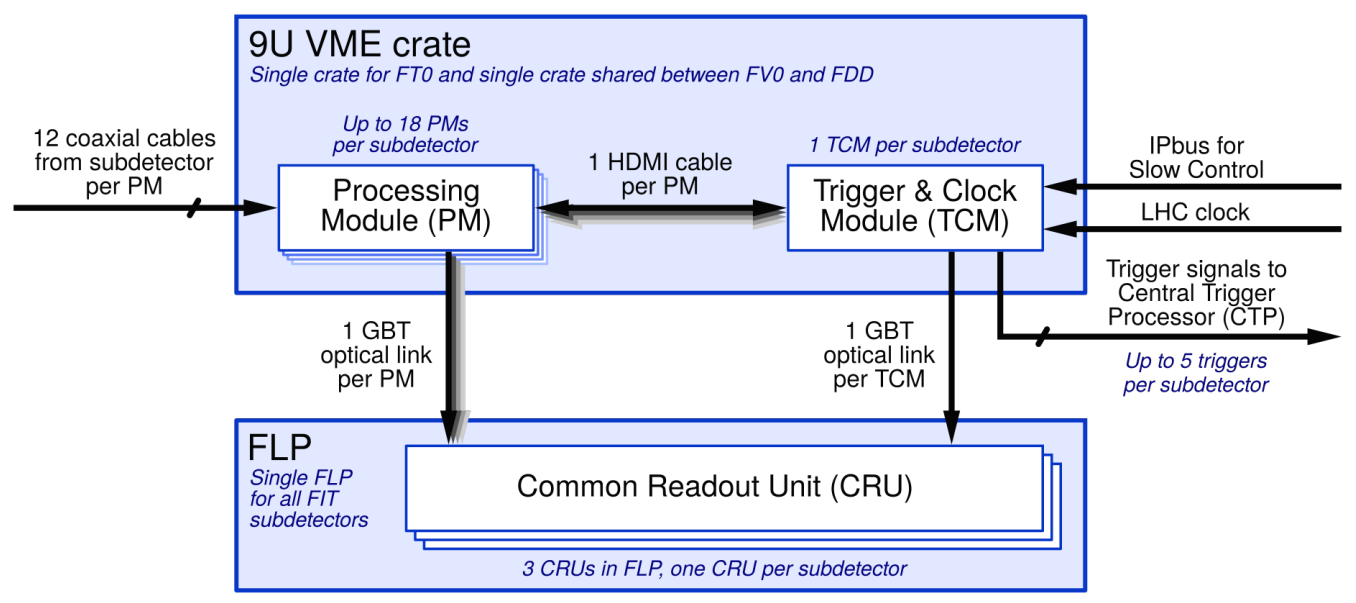

Figure 2: Scheme of the FIT FEE.

The scheme of FIT FEE is shown in Fig. 2. It is a combination of just two modules: a Processing Module (PM) and a Trigger and Clock Module (TCM). Each subdetector uses one TCM and, depending on the number of channels, multiple PM modules. FT0 will use 18 PMs, FV0 will use 6 PMs, and FDD will use 2 PMs. TCM modules keep the PM modules synchronized by providing an interface to the LHC clock. Each of the 12 PM inputs is equipped with a fan-out amplifier leading to a constant fraction discriminator (CFD) and an analogue-to-digital converter (ADC). The CFD generates the ADC gate and feeds the signal arrival time to a time-to-digital converter (TDC). Signal charge is obtained by digital integration of the ADC output by an on-board, FPGA programmable, element. Since the ADC readout is relatively slow, each channel uses two ADCs working sequentially to reduce the dead time. The digitized time and charge are sent, via a GBT optical link, to a dedicated PC, called First Level Processor (FLP), for further processing. In parallel, each PM unit evaluates the data from its 12 inputs and sends this pre-trigger information to the TCM. The TCM combines them to generate the final triggers.

\section{Test results}

The design details and results of tests of several FIT detector prototypes have already been published before (see [2-6]), as well as the simulated detector performance (see [7, 8]). The focus of this paper is on the final FEE and its response to signals from real detectors. Three detector modules 

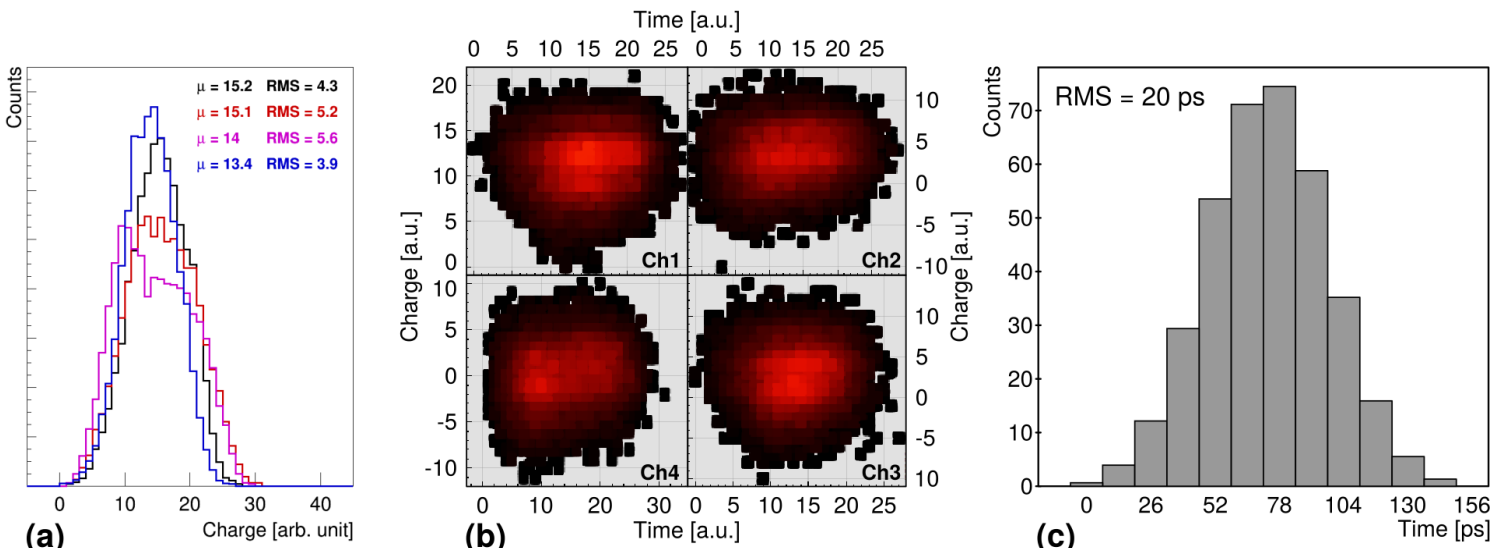

Figure 3: Performance of the FEE connected to the partly assembled FT0-A detector using laser as signal source: (a) charge distribution of 4 random channels, (b) correlation between time and charge, and (c) time resolution of the generated trigger.

have been installed inside the actual FT0-A frame. The signals originating from picosecond laser pulses were delivered using an integrated laser calibration system. The objectives and results of the tests can be summarized as follows.

\section{- Crosstalk}

Once installed in the FT0-A frame, up to 12 signal cables and 3 high-voltage cables from three sensors are routed parallel and at very close proximity to one another. The crosstalk related to the proximity of cables was measured to be negligible.

\section{- FEE channel uniformity in terms of charge and time}

An example of charge distribution of 4 channels, in response to laser pulses with intensity corresponding to 1-MIP signal, is shown in Fig. 3 (a). The charge uniformity is acceptable. The uniformity of channel response in terms of time was also measured and found equally adequate.

\section{- Slewing}

Figure 3 (b) shows that there is no correlation between time and amplitude for low-intensity laser pulses corresponding to 1-MIP signal, which means the CFD integrated in the PM board works as expected.

\section{- Time resolution of the vertex trigger}

Two PMs and one TCM were used to test the trigger generation. One PM represented the $\mathrm{A}$-side detector and the other the $\mathrm{C}$-side detector. The vertex trigger requires a coincidence of signals from A- and C-side in a narrow time gate. The trigger time distribution plot presented in Fig. 3 (c) shows the vertex trigger time RMS of 20 ps. It is a conservative result because only three detector channels were active in this test: one in one PM and two in the other PM. Moreover, the laser intensity was set to only 1.3-MIP equivalent per active channel. Such conditions correspond to the most challenging low-multiplicity pp collisions. 
Table 1: Status and schedule of FIT subdetectors as of Dec. 8, 2020.

\begin{tabular}{cccc}
\hline Subdetector & Assembly status & Standalone testing & Installation schedule \\
\hline FT0-C & $\begin{array}{c}\text { Fully assembled } \\
\text { To be scheduled } \\
\text { FT0-A }\end{array}$ & Done & installed \\
FV0 & Fully assembled & Pending & May 2021 \\
FDD (A \& C) & $\begin{array}{c}\text { Ongoing } \\
\text { (ready by Jan 2021) }\end{array}$ & tests: Jan - Mar 2021) & May 2021 \\
\hline
\end{tabular}

\section{Construction and installation status}

Table 1 presents the status and installation schedule of each FIT detector. Notably, the installation schedule changes rapidly together with the uncertain global situation prevalent in 2020, that affects travel and work conditions.

\section{Conclusions}

The new Fast Interaction Trigger is an essential part of the upgrade of ALICE. In the online mode, FIT will generate an interaction trigger, monitor luminosity, provide initial vertex position and measure forward multiplicity. In the offline mode, it will deliver the precise collision time for the TOF-based particle identification, yield the centrality and event plane, and measure cross sections of diffractive processes. To deliver the required functionalities, FIT incorporates three different detector technologies with a common FEE. As of December 2020, the construction, installation and commissioning of FIT is proceeding as planned. The FT0-C is already installed. The installation of FDD is due in February 2021 and the one of the FV0 and FT0-A is scheduled in May 2021. The global commissioning of ALICE will start in July 2021.

\section{References}

[1] B. Abelev, (ALICE Collaboration). Upgrade of the ALICE Experiment: Letter Of Intent. J. Phys., G41:087001, 2014.

[2] Yu.A. Melikyan et al. Performance of the cost-effective Planacon ${ }^{\circledR}$ MCP-PMTs in strong magnetic fields. Nucl. Instrum. Meth. A, 983:164591, 2020.

[3] Yu.A. Melikyan. Performance of Planacon MCP-PMT photosensors under extreme working conditions. Nucl. Instrum. Meth. A, 952:161689, 2020.

[4] Varlen Grabski. New fiber read-out design for the large area scintillator detectors: providing good amplitude and time resolutions, 2019. arXiv:1909.01184.

[5] E. V. Antamanova et al. Anode current saturation of ALD-coated Planacon® MCP-PMTs. JINST, 13(09):T09001, 2018.

[6] D.A. Finogeev et al. Performance study of the fast timing Cherenkov detector based on a microchannel plate PMT. J. Phys. Conf. Ser., 798(1):012168, 2017.

[7] A. I. Maevskaya. Fast Interaction Trigger for the upgrade of the ALICE experiment at CERN: design and performance. EPJ Web Conf., 204:11003, 2019.

[8] Wladyslaw Henryk Trzaska. New Fast Interaction Trigger for ALICE. Nucl. Instrum. Meth., A845:463-466, 2017. 